\title{
Association of Lipoprotein (a) and Coronary Artery Lesion and In-hospital Outcomes in Patients with Acute Coronary Syndrome Undergoing Percutaneous Coronary Intervention
}

\section{Baoquan Wu}

Chinese Academy of Medical Sciences \& Peking Union Medical College Fuwai Hospital Hanjun Zhao

Chinese Academy of Medical Sciences \& Peking Union Medical College Fuwai Hospital Changhua Liu

Chinese Academy of Medical Sciences \& Peking Union Medical College Fuwai Hospital Hao Lu

Chinese Academy of Medical Sciences \& Peking Union Medical College Fuwai Hospital

\section{Ruishuang Liu}

Chinese Academy of Medical Sciences \& Peking Union Medical College Fuwai Hospital Juan Long

Chinese Academy of Medical Sciences \& Peking Union Medical College Fuwai Hospital

\section{Zhiling Zhang}

Chinese Academy of Medical Sciences \& Peking Union Medical College Fuwai Hospital

Fanfang Zeng ( $\nabla$ zengfanfang123@tom.com )

Shenzhen Sun Yat-sen Cardiovascular Hospital

\section{Research}

Keywords: acute coronary syndrome, lipoprotein (a), coronary artery lesion, clinical outcomes

Posted Date: June 19th, 2020

DOI: https://doi.org/10.21203/rs.3.rs-36415/v1

License: (c) (i) This work is licensed under a Creative Commons Attribution 4.0 International License. Read Full License 


\section{Abstract}

Background: Current study was to evaluate association of Lipoprotein (a) [Lp(a)] and coronary artery lesion and in-hospital outcomes in acute coronary syndrome (ACS) patients undergoing percutaneous coronary intervention (PCl).

Methods: Baseline characteristics, characteristics of coronary artery lesion, medications use, and cardiovascular events during hospitalization were collected. Based on $L p(a)$ level, patients were divided into low $(<30 \mathrm{mg} / \mathrm{dL})$ and high ( $\geq 30 \mathrm{mg} / \mathrm{dL})$ groups.

Results: Compared to those with low $L p(a)$, patients with high $L p(a)$ had larger numbers of coronary arteries $\geq 70 \%$ stenosis and longer coronary artery lesion $(P<0.05)$. Patients with high $L p(a)$ were more likely to have left anterior descending artery lesion, pre-PCI TIMI flow grade 0 and post-PCI TIMI flow grade 2 , and type $\mathrm{C}$ coronary lesion $(\mathrm{P}<0.05)$. After adjustment, high $\mathrm{Lp}(\mathrm{a})$ remained associated with higher odds of having coronary artery $\geq 70 \%$ stenosis, type $\mathrm{C}$ coronary lesion and pre-PCI TIME flow grade $1 / 0$. Compared to those with low $L p(a)$, patients with high $L p(a)$ had a higher unadjusted odds of acute stent thrombosis (odds ratio [OR] 1.10 and 95\% confidence interval [CI] 1.01-2.27), congestive heart failure (OR 1.24 and 95\% $\mathrm{Cl} 1.15-2.38$ ) and composite in-hospital outcomes (OR 1.28 and 95\% $\mathrm{Cl} 1.18$ 2.42). After adjustment, patients with high $L p(a)$ remained had a higher odds of congestive heart failure (OR 1.08 and 95\% $\mathrm{Cl} 1.01-1.78$ ) and composite in-hospital outcomes (OR 1.12 and 95\% Cl 1.04-1.81).

Conclusion: High Lp(a) was associated with more severe coronary artery lesion, and higher risk of congestive heart failure and composite in-hospital outcomes.

\section{Background}

Coronary heart disease (CHD) remains a leading cause of morbidity and mortality worldwide despite advancements in invasive and medication treatments have been achieved in the past three decades [13]. Dyslipidemia is a major risk factor for $\mathrm{CHD}$ and lowering serum low-density lipoprotein cholesterol (LDL-C) level with statins treatment is beneficial to reduce cardiovascular events after percutaneous coronary intervention $(\mathrm{PCl})$ treatment $[3,4]$. However, numerous studies have shown that despite receiving intensive statins treatment, a substantial proportion of patients remain experience cardiovascular events including myocardial infarction, stent thrombosis and cardiovascular death, indicative of a significant residual cardiovascular risk in these populations [5-8].

Traditional therapies such as statins targeting LDL-C have been unsatisfactory, and many studies found that increased lipoprotein (a) [ $L p(a)$ ] level was associated with cardiovascular events [9-12]. $L p(a)$ is a LDL-like particle synthesized in the liver [13]. Serum Lp(a) level is predominantly dependent on the number of repeats in the kringle IV type 2 protein domain [13]. Numerous studies have shown that increased serum Lp(a) level portends a higher cardiovascular risk [9-12]. Unfortunately, statins treatment has no effects on $L p(a)$ reduction $[13,14]$. Of note, $L p(a)$ gene expression varies substantially between different racial/ethnic groups $[15,16]$, and results of prior observational and epidemiological studies are 
almost from Caucasian populations. How are the effects of increased serum Lp(a) level on clinical outcomes in Chinese Han populations with acute coronary syndrome (ACS) have not been fully elucidated yet. In last several years, accumulating evidence have consistently shown that Lp(a) could be reduced with PCSK9 inhibitor, which in turn was associated with a lower incidence of cardiovascular events $[17,18]$. Therefore, $L p(a)$ is increasingly recognized as a modifiable cardiovascular risk factor currently, and it is speculated that $L p(a)$ reduction may further reduce residual cardiovascular risk.

Herein, using a retrospective design, we collected data of ACS patients undergoing $\mathrm{PCl}$ in our hospital, and the aims of current analysis was to evaluate: 1 ) the association of $L p(a)$ and coronary artery lesion, 2) the association of Lp(a) and in-hospital outcomes.

\section{Methods}

\section{Study participants}

Current study was approved by the Institution Review Board of our hospital and since this was a retrospective study, no written consent form was required. Patients who were diagnosed as CHD between January of 2017 and December of 2019 were screened, and the inclusion criteria were as follows: $\geq$ 18 years old; an admission diagnosis of ACS; undergoing PCl; received intensive statins treatment during hospitalization; and had Lp(a) measurement at admission. The exclusion criteria were as follows: undergoing coronary artery bypass grafting; critically ill patients that required mechanical circulatory support or ventilation; failed to have coronary artery stenting; or died before $\mathrm{PCl}$ (Fig. 1).

\section{Data collection}

All data were collected from electronic health record by three independent investigators. Data, including vital signs (blood pressure and heart rate at admission), anthropometrics (height and weight), demographics (age and sex), socioeconomic status (highest educational attainment), cardiovascular risk factor (smoking status and obesity), comorbidities (hypertension, diabetes mellitus, dyslipidemia, atrial fibrillation, congestive heart failure, $\mathrm{CHD}$, prior myocardial infarction, prior $\mathrm{PCl}$, prior coronary artery bypass grafting, ischemic stroke/transient ischemic stroke (TIA), peripheral vascular disease [PVD] and chronic kidney disease [CKD]), were collected. Body mass index (BMI) was calculated using weight in kilograms divided by height in squared meters and $\mathrm{BMI} \geq 28 \mathrm{~kg} / \mathrm{m}^{2}$ was defined as obesity in accordance to the WHO criterion for Asian populations. Laboratory parameters included lipid panel, glycated hemoglobin A1c (HbA1c), serum levels of creatinine, C-reactive protein (CRP), high-sensitivity cardiac troponin-I (Hs-cTNI), and N-terminal pro-B natriuretic peptide (NT-proBNP). Creatinine was used to calculate estimated glomerular filtration rate (eGFR). Procedural characteristics included duration of symptom onset to undergo $\mathrm{PCl}$, arterial access site, number of coronary arteries $\geq 70 \%$ stenosis, lesion location and lesion length, TIMI flow grade of pre- and post-PCI treatment, minimum lumen diameter of pre- and post-PCI treatment in the lesion vessel, type and number of stent implanted, volume of contrast 
and antiplatelet loading used during PCl. Medication use before admission were reconciled by physicians and medication use during hospitalization was extracted from electronic health record. The SYNTAX score was calculated by an independent interventional cardiologist using dedicated software (www.SYNTAXscore.com). All these data were entered to the encrypted excel dataset.

\section{In-hospital outcomes}

In-hospital cardiovascular events were identified and adjudicated by independent cardiologists who did not involve in the current study. All the cardiovascular events were adjudicated based on clinical symptoms/signs, laboratory tests, and imagining studies rather than based on ICD-9 code, and the cardiovascular events included acute stent thrombosis, myocardial infarction, ischemic stroke/TIA, congestive heart failure and cardiovascular mortality. In specific, acute stent thrombosis was diagnosed based on coronary angiography with evidence of thrombotic occlusion of the implanted coronary stent and all the diagnosis was definite acute stent thrombosis based on the Academic Research Consortium criteria [19]. Myocardial infarction was diagnosed based on clinical symptoms (e.g. substernal chest pain), electrocardiographic exam (e.g. ST-segment elevation or depression) and plasma concentration of cardiac troponin I above the 99th percentile upper normal limit [20]. Patients with MI due to acute stent thrombosis was defined as the event of acute stent thrombosis. Ischemic stroke/TIA was diagnosed based on neurologic deficits plus cerebrovascular imaging evidence (e.g. computed tomography) [21]. Congestive heart failure was diagnosed by clinical manifestations including cardiac dyspnea, pink frothy sputum, and crackles in bilateral lung as well as a supporting examination such as echocardiogram or chest X-ray [22]. Cardiovascular mortality was defined as death due to cardiac etiologies.

\section{Statistical analysis}

Continuous variables were presented as mean \pm standard deviation (SD) or median (interquartile range; IQR), and categorical variables were presented as number and frequency. Participants were divided into low $L p(a)(<30 \mathrm{mg} / \mathrm{dL}$ ) and high $\mathrm{Lp}(\mathrm{a})(\geq 30 \mathrm{mg} / \mathrm{dL})$ groups based on recommendation [23]. Betweengroup differences were evaluated by Student $t$ test or Mann-Whitney-Wilcoxon text for continuous variables, and chi-squared test for categorial variables. Low Lp(a) group was served as the reference group in unadjusted and adjusted models. Logistic regression analysis was performed to evaluate the association of $\mathrm{Lp}(\mathrm{a})$ and coronary artery lesion and in-hospital outcomes with adjustment for potential covariates. In specific, in the regression model, age, gender and covariates that were significantly different at baseline were included.

Since the SYNTAX score was included in regression model, and in order to avoid collinearity, the individual component of coronary lesion (e.g. number of arteries $\geq 70 \%$ stenosis) was not included in the regression model. All analyses were conducted using the SPSS 23.0 statistical software and a two-sided $P$ value $<0.05$ was considered as statistical significance. 


\section{Results}

\section{Baseline characteristics comparisons}

Baseline characteristics comparisons were shown in Table 1. Compared to those with low Lp(a), patients with high $\mathrm{Lp}(\mathrm{a})$ were more likely to be men; had higher BMI and higher prevalence of diabetes mellitus and dyslipidemia $(P<0.05)$. Serum levels of $L p(a), H s-c T N I$ and NT-proBNP were higher, while eGFR was lower in high $L p(a)$ group $(P<0.05)$. 
Table 1

Baseline characteristics comparisons

\begin{tabular}{|c|c|c|c|}
\hline Variables & $\begin{array}{l}\mathrm{Lp}(\mathrm{a})<30 \mathrm{mg} / \mathrm{dL} \\
(\mathrm{n}=566)\end{array}$ & $\begin{array}{l}\mathrm{Lp}(\mathrm{a}) \geq 30 \mathrm{mg} / \mathrm{dL} \\
(\mathrm{n}=726)\end{array}$ & P-value \\
\hline Age (years) & $55.9 \pm 11.8$ & $57.1 \pm 13.2$ & 0.87 \\
\hline Men, n (\%) & $355(62.7)$ & $540(74.4)$ & $<0.001$ \\
\hline$\geq$ High school, n (\%) & $337(59.5)$ & $412(56.7)$ & 0.63 \\
\hline Systolic blood pressure $(\mathrm{mm} \mathrm{Hg})$ & $128 \pm 14$ & $130 \pm 16$ & 0.41 \\
\hline Diastolic blood pressure $(\mathrm{mm} \mathrm{Hg})$ & $73 \pm 10$ & $75 \pm 11$ & 0.33 \\
\hline Heart rate (beat per minute) & $86 \pm 19$ & $88 \pm 18$ & 0.19 \\
\hline Body mass index $\left(\mathrm{kg} / \mathrm{m}^{2}\right)$ & $24.1 \pm 6.3$ & $25.7 \pm 6.6$ & 0.007 \\
\hline Current smoker, n (\%) & $248(43.8)$ & $315(43.4)$ & 0.28 \\
\hline Obesity, n (\%) & $175(30.9)$ & $238(32.9)$ & 0.75 \\
\hline Hypertension, n (\%) & $382(67.5)$ & $475(65.4)$ & 0.69 \\
\hline Diabetes mellitus, n (\%) & $163(28.8)$ & $251(34.6)$ & $<0.001$ \\
\hline Dyslipidemia, n (\%) & $278(49.1)$ & $398(54.8)$ & $<0.001$ \\
\hline Atrial fibrillation, n (\%) & $52(9.2)$ & $75(10.3)$ & 0.90 \\
\hline Congestive heart failure, $\mathrm{n}(\%)$ & $63(11.1)$ & $81(11.2)$ & 0.98 \\
\hline Coronary heart disease, n (\%) & $118(20.8)$ & $166(22.9)$ & 0.62 \\
\hline Prior myocardial infarction, $\mathrm{n}(\%)$ & $60(10.6)$ & $85(11.7)$ & 0.80 \\
\hline Prior PCl, n (\%) & $82(14.5)$ & $102(14.0)$ & 0.93 \\
\hline Prior CABG, n (\%) & $9(1.6)$ & $13(1.8)$ & 0.77 \\
\hline Ischemic stroke/TIA, n (\%) & $38(6.7)$ & $55(7.6)$ & 0.38 \\
\hline Peripheral vascular disease, $\mathrm{n}(\%)$ & $30(5.3)$ & $49(6.7)$ & 0.50 \\
\hline Chronic kidney disease, n (\%) & $78(13.4)$ & $104(14.3)$ & 0.68 \\
\hline $\mathrm{HbA} 1 \mathrm{c}(\%)$ & $5.8 \pm 0.5$ & $5.9 \pm 0.6$ & 0.91 \\
\hline Total cholesterol (mmol/L) & $4.9 \pm 0.9$ & $5.0 \pm 1.1$ & 0.55 \\
\hline
\end{tabular}

$\mathrm{PCl}$, percutaneous coronary intervention; $\mathrm{CABG}$, coronary artery bypass grafting; $\mathrm{TIA}$, transient ischemic attack; HbA1c, glycated hemoglobin A1 c; LDL-C, low-density lipoprotein cholesterol; HDL-C, high density lipoprotein cholesterol; NT-proBNP, N-terminal pro-B type natriuretic peptide; Hs-CTnl, high sensitivity cardiac troponin-l; eGFR, estimated glomerular filtration rate 


\begin{tabular}{|c|c|c|c|}
\hline Variables & $\begin{array}{l}\mathrm{Lp}(\mathrm{a})<30 \mathrm{mg} / \mathrm{dL} \\
(\mathrm{n}=566)\end{array}$ & $\begin{array}{l}\mathrm{Lp}(\mathrm{a}) \geq 30 \mathrm{mg} / \mathrm{dL} \\
(\mathrm{n}=726)\end{array}$ & P-value \\
\hline LDL-C (mmol/L) & $3.0 \pm 0.5$ & $3.1 \pm 0.5$ & 0.49 \\
\hline $\mathrm{HDL}-\mathrm{C}(\mathrm{mmol} / \mathrm{L})$ & $1.2 \pm 0.4$ & $1.1 \pm 0.4$ & 0.73 \\
\hline Triglyceride (mmol/L) & $1.8(0.7-2.7)$ & $1.9(0.6-2.8)$ & 0.16 \\
\hline Lipoprotein (a) (mg/dL) & $17.6(10.5-26.8)$ & $73.6(41.4-137.9)$ & $<0.001$ \\
\hline C-reactive protein $(\mathrm{mg} / \mathrm{dL})$ & $6.9 \pm 2.0$ & $8.2 \pm 2.3$ & 0.03 \\
\hline NT-proBNP (pg/mL) & 366.7 (142.8-632.2) & $394.2(170.9-756.4)$ & 0.01 \\
\hline $\mathrm{Hs}-\mathrm{CTnl}(\mathrm{ng} / \mathrm{mL})$ & $7.3 \pm 3.1$ & $9.5 \pm 3.3$ & 0.009 \\
\hline Creatinine (umol/L) & $96.4 \pm 18.5$ & $97.1 \pm 19.2$ & 0.48 \\
\hline eGFR $\left(\mathrm{ml} / \mathrm{min} / 1.73 \mathrm{~m}^{2}\right)$ & $69.6 \pm 13.6$ & $67.3 \pm 11.8$ & 0.004 \\
\hline \multicolumn{4}{|c|}{$\begin{array}{l}\mathrm{PCl} \text {, percutaneous coronary intervention; } \mathrm{CABG} \text {, coronary artery bypass grafting; TIA, transient } \\
\text { ischemic attack; HbA1c, gly cated hemoglobin A1c; LDL-C, low-density lipoprotein cholesterol; HDL-C, } \\
\text { high density lipoprotein cholesterol; NT-proBNP, N-terminal pro-B type natriuretic peptide; Hs-CTnl, high } \\
\text { sensitivity cardiac troponin-l; eGFR, estimated glomerular filtration rate }\end{array}$} \\
\hline
\end{tabular}

\section{Procedural characteristics comparisons}

As shown in Table 2, compared to those with low $L p(a)$, patients with high $L p(a)$ had larger numbers of coronary arteries $\geq 70 \%$ stenosis and had longer coronary artery lesion $(P<0.05)$. In addition, patients with high $\mathrm{Lp}(\mathrm{a})$ were more likely to have left anterior descending artery lesion, pre-PCI TIMI flow grade 0 and post-PCI TIMI flow grade 2 , and type $\mathrm{C}$ coronary lesion $(\mathrm{P}<0.05)$. The use of glycoprotein Ilb/llla inhibitor during $\mathrm{PCl}$ was also higher in patients with high $\mathrm{Lp}(\mathrm{a})(\mathrm{P}<0.05)$. In addition, the SYNTAX score was also higher in patients with high versus low $L p(a)(21.2 \pm 5.7$ vs $17.7 \pm 4.6)$. No differences in other procedural characteristics were observed. 
Table 2

Procedural characteristics comparisons

\begin{tabular}{|c|c|c|c|}
\hline Variables & $\begin{array}{l}\mathrm{Lp}(\mathrm{a})<30 \mathrm{mg} / \mathrm{dL} \\
(\mathrm{n}=566)\end{array}$ & $\begin{array}{l}\mathrm{Lp}(a) \geq 30 \mathrm{mg} / \mathrm{dL} \\
(\mathrm{n}=726)\end{array}$ & P-value \\
\hline Duration of symptom onset to $\mathrm{PCl}$ (day) & $1.9(0.5-2.8)$ & $2.0(0.6-2.9)$ & 0.37 \\
\hline ST-segment elevation MI, n (\%) & $178(31.4)$ & $237(32.6)$ & 0.44 \\
\hline Non-ST-segment elevation MI, n (\%) & $157(27.7)$ & $204(28.1)$ & 0.61 \\
\hline Unstable angina, n (\%) & $231(40.9)$ & $285(39.3)$ & 0.19 \\
\hline Femoral artery access, n (\%) & $265(46.8)$ & $343(47.2)$ & 0.53 \\
\hline Number of arteries $\geq 70 \%$ stenosis & $1.4 \pm 0.5$ & $1.6 \pm 0.6^{*}$ & 0.008 \\
\hline Lesion length, mm & $25.3 \pm 6.5$ & $27.6 \pm 6.8^{*}$ & 0.01 \\
\hline \multicolumn{4}{|l|}{ Lesion locations } \\
\hline Left main, $n(\%)$ & $89(15.7)$ & $116(16.0)$ & 0.72 \\
\hline LAD, n (\%) & $286(50.5)$ & $450(62.0)^{\star}$ & $<0.001$ \\
\hline LCX, n (\%) & $198(35.0)$ & $275(37.9)$ & 0.36 \\
\hline RCA, n (\%) & $247(43.6)$ & $308(42.4)$ & 0.22 \\
\hline \multicolumn{4}{|l|}{ Pre-PCI TIMI flow grade } \\
\hline Grade 3 & 0 & 0 & - \\
\hline Grade 2 & 0 & 0 & - \\
\hline Grade 1 & $369(65.2)$ & $427(58.8)$ & 0.71 \\
\hline Grade 0 & $197(34.8)$ & $299(41.2)^{\star}$ & 0.03 \\
\hline \multicolumn{4}{|l|}{ Post-PCI TIMI flow grade } \\
\hline Grade 3 & $543(95.9)$ & $703(90.4)$ & 0.92 \\
\hline Grade 2 & $23(4.1)$ & $70(9.6)^{\star}$ & 0.006 \\
\hline Grade 1 & 0 & 0 & - \\
\hline Grade 0 & 0 & 0 & - \\
\hline Pre-PCl minimum lumen diameter, $\mathrm{mm}$ & $0.78 \pm 0.12$ & $0.76 \pm 0.10$ & 0.28 \\
\hline
\end{tabular}

$\mathrm{PCl}$, percutaneous coronary intervention; MI, myocardial infarction; LAD, left anterior descending; LCX, left circumflex; RCA, right coronary artery; * $\mathrm{P}<0.05$ versus $L p(a)<30 \mathrm{mg} / \mathrm{dL}$ group 


\begin{tabular}{|c|c|c|c|}
\hline Variables & $\begin{array}{l}\mathrm{Lp}(\mathrm{a})<30 \mathrm{mg} / \mathrm{dL} \\
(\mathrm{n}=566)\end{array}$ & $\begin{array}{l}\mathrm{Lp}(a) \geq 30 \mathrm{mg} / \mathrm{dL} \\
(\mathrm{n}=726)\end{array}$ & P-value \\
\hline Post $-\mathrm{PCl}$ minimum lumen diameter, $\mathrm{mm}$ & $2.32 \pm 0.63$ & $2.31 \pm 0.61$ & 0.85 \\
\hline Type C coronary lesion, $\mathrm{n}(\%)$ & $138(24.4)$ & $245(33.7)$ & 0.07 \\
\hline Antiplatelet medication loading, $\mathrm{n}(\%)$ & $524(92.6)$ & $674(92.8)$ & 0.69 \\
\hline Glycoprotein Ilb/Illa inhibitor, n (\%) & $40(7.1)$ & $89(12.3)^{\star}$ & 0.03 \\
\hline Volume of contrast (ml) & $164.5 \pm 39.6$ & $162.7 \pm 40.3$ & 0.54 \\
\hline Number of stents implanted & $1.8 \pm 0.6$ & $1.9 \pm 0.7$ & 0.96 \\
\hline Drug-eluting stent, $\mathrm{n}(\%)$ & $548(96.8)$ & $704(97.0)$ & 0.80 \\
\hline SYNTAX score & $17.7 \pm 4.6$ & $21.2 \pm 5.7^{*}$ & 0.002 \\
\hline
\end{tabular}

\section{Comparisons of medication use at admission and during hospitalization}

As presented in Table 3, the use of statins and anti-diabetes were higher in patients with high versus low $L p(a)(P<0.05)$. During hospitalization, compared to admission, the use of guideline recommended medication was significantly increased in both groups. The use of ticagrelor, anti-diabetes and low molecular-weight heparin was also higher in patients with high $\operatorname{Lp}(a)(P<0.05)$. 
Table 3

Medications use at admission and during hospitalization

\begin{tabular}{|c|c|c|}
\hline Medications & $\operatorname{Lp}(a)<30 \mathrm{mg} / \mathrm{dL}(\mathrm{n}=566)$ & $\mathrm{Lp}(\mathrm{a}) \geq 30 \mathrm{mg} / \mathrm{dL}(\mathrm{n}=726)$ \\
\hline \multicolumn{3}{|l|}{ At admission } \\
\hline Aspirin, n (\%) & $303(53.5)$ & $397(54.7)$ \\
\hline Clopidogrel, n (\%) & $37(6.5)$ & $50(6.9)$ \\
\hline Ticagrelor, n (\%) & $12(2.1)$ & $16(2.2)$ \\
\hline Statins, n (\%) & $249(44.0)$ & $368(50.7)^{\star}$ \\
\hline Beta-blocker, n (\%) & $231(40.8)$ & $302(41.6)$ \\
\hline ACEI/ARB, n (\%) & $285(50.4)$ & $384(82.9)$ \\
\hline Calcium channel blocker, n (\%) & $186(32.9)$ & $243(33.5)$ \\
\hline Anti-diabetes, n (\%) & $134(23.7)$ & $232(32.0)^{\star}$ \\
\hline \multicolumn{3}{|l|}{ During hospitalization } \\
\hline Aspirin, n (\%) & $566(100)$ & $726(100)$ \\
\hline Clopidogrel, n (\%) & $518(91.5)$ & $626(86.2)$ \\
\hline Ticagrelor, n (\%) & $48(8.5)$ & $100(13.8)^{\star}$ \\
\hline Statins, n (\%) & $538(95.1)$ & $687(94.6)$ \\
\hline Beta-blocker, n (\%) & $465(82.3)$ & $602(82.9)$ \\
\hline ACEI/ARB, n (\%) & $421(74.4)$ & $530(73.0)$ \\
\hline Calcium channel blocker, n (\%) & $134(23.7)$ & $183(25.2)$ \\
\hline Anti-diabetes, n (\%) & $162(25.1)$ & $246(33.9)^{\star}$ \\
\hline Low molecular-weight heparin, n (\%) & $34(6.0)$ & $74(10.2)^{\star}$ \\
\hline \multicolumn{3}{|c|}{ ACEI/ARB, angiotensin-converting enzyme inhibitor/angiotensin receptor blocker; } \\
\hline * $\mathrm{P}<0.05$ versus $L p(a)<30 \mathrm{mg} / \mathrm{dL} \mathrm{g}$ & & \\
\hline
\end{tabular}

\section{Association of $L p(a)$ and coronary artery lesion}

Association of $L p(a)$ level and coronary artery lesion were evaluated and shown in Table 4. In reference to low $L p(a)$, high $L p(a)$ was associated with higher unadjusted odds of having coronary artery $\geq 70 \%$ stenosis, type $\mathrm{C}$ coronary lesion, and pre- and post-PCI TIME flow grade 1/0. After adjusted for potential covariates, high $L p(a)$ remained associated with higher odds of having coronary artery $\geq 70 \%$ stenosis, 
type $\mathrm{C}$ coronary lesion and pre-PCI TIME flow grade $1 / 0$. It is noted that additionally adjusted for glycoprotein Ilb/Illa inhibitor use during $\mathrm{PCl}$, high $\mathrm{Lp}(\mathrm{a})$ was associated with higher odds of having post$\mathrm{PCI}$ TIME flow grade $1 / 0$, suggesting that the use of glycoprotein Ilb/llla inhibitor might attenuate the adverse effect of $L p(a)$.

Table 4

Association of $L p(a)$ and coronary artery lesion

$\begin{array}{lcc}\text { High Lp(a) versus low Lp(a) } & \text { Odds ratio } & \text { 95\% Confid } \\ \text { Number of arteries } \geq 70 \% \text { stenosis } & & \\ \text { Unadjusted } & 1.95 & 1.46-2.84 \\ \text { Adjusted } & 1.34 & 1.09-2.01 \\ \text { Type C coronary lesion } & & \\ \text { Unadjusted } & 1.67 & 1.34-2.22 \\ \text { Adjusted } & 1.24 & 1.09-1.83\end{array}$

Pre-PCI TIMI flow grade 1/0

$\begin{array}{lcc}\text { Unadjusted } & 1.63 & 1.30-2.18 \\ \text { Adjusted } & 1.22 & 1.04-1.72\end{array}$

Post-PCI TIMI flow grade 1/0

Unadjusted

Adjusted

Additionally adjusted to glycoprotein IIb/IIla inhibitor

Adjusted for age, gender, smoking status, hypertension, diabetes mellitus, dyslipidemia, C-reactive protein, estimated glomerular filtration rate, and statins use at admission

\section{Association of $\operatorname{Lp}(\mathrm{a})$ and in-hospital outcomes}

As shown in Table 5, compared to patients with low $L p(a)$, patients with high $L p(a)$ had a higher unadjusted odds of acute stent thrombosis (odds ratio [OR] 1.10 and 95\% confidence interval [Cl] 1.012.27), congestive heart failure (OR 1.24 and $95 \% \mathrm{Cl} 1.15-2.38$ ) and composite in-hospital outcomes (OR 1.28 and $95 \% \mathrm{Cl} 1.18-2.42$ ). After adjustment for potential covariates, patients with high Lp(a) remained had a higher odds of congestive heart failure (OR 1.08 and $95 \% \mathrm{Cl} 1.01-1.78$ ) and composite in-hospital outcomes (OR 1.12 and $95 \% \mathrm{Cl} 1.04-1.81$ ). 
Table 5

Association of $L p(a)$ and in-hospital outcomes

\begin{tabular}{|c|c|c|c|c|}
\hline Cardiovascular events & $\begin{array}{l}\operatorname{Lp}(a)<30 \mathrm{mg} / \mathrm{dL} \\
(\mathrm{n}=566)\end{array}$ & $\begin{array}{l}\operatorname{Lp}(a) \geq 30 \mathrm{mg} / \mathrm{dL} \\
(\mathrm{n}=726)\end{array}$ & Unadjusted & Adjusted \\
\hline & \multicolumn{2}{|l|}{$\mathrm{n}(\%)$} & \multicolumn{2}{|c|}{$\begin{array}{l}\text { Odds ratio ( } 95 \% \text { confidence } \\
\text { interval) }\end{array}$} \\
\hline $\begin{array}{l}\text { Acute stent } \\
\text { thrombosis, n (\%) }\end{array}$ & $4(0.7)$ & $12(1.7)$ & $\begin{array}{l}1.10(1.01- \\
2.27)\end{array}$ & $\begin{array}{l}1.01(0.78- \\
1.70)\end{array}$ \\
\hline $\begin{array}{l}\text { Myocardial infarction, } \\
\text { n (\%) }\end{array}$ & $15(2.7)$ & $32(4.4)$ & $\begin{array}{l}1.16(0.94- \\
2.25)\end{array}$ & $\begin{array}{l}1.05(0.83- \\
1.57)\end{array}$ \\
\hline \multirow{2}{*}{$\begin{array}{l}\text { Ischemic stroke/TIA, n } \\
(\%)\end{array}$} & $6(1.1)$ & $10(1.4)$ & \multirow{2}{*}{$\begin{array}{l}1.19(0.90- \\
2.23)\end{array}$} & $\begin{array}{l}1.03(0.80- \\
1.74)\end{array}$ \\
\hline & $54(9.5)$ & $94(12.9)$ & & \multirow{2}{*}{$\begin{array}{l}1.08(1.01- \\
1.78)\end{array}$} \\
\hline $\begin{array}{l}\text { Congestive heart } \\
\text { failure, } \mathrm{n}(\%)\end{array}$ & $10(1.8)$ & $14(1.9)$ & $\begin{array}{l}1.24(1.15- \\
2.38)\end{array}$ & \\
\hline $\begin{array}{l}\text { Cardiovascular } \\
\text { mortality, n (\%) }\end{array}$ & & & $\begin{array}{l}1.08(0.84- \\
2.11)\end{array}$ & $\begin{array}{l}1.00(0.65- \\
1.42)\end{array}$ \\
\hline Composite, n (\%) & $89(15.7)$ & $162(22.3)$ & $\begin{array}{l}1.28(1.18- \\
2.42)\end{array}$ & $\begin{array}{l}1.12(1.04- \\
1.81)\end{array}$ \\
\hline \multicolumn{5}{|c|}{$\begin{array}{l}\text { TIA, transient ischemic attack; Adjusted for age, gender, body mass index, diabetes mellitus, } \\
\text { dyslipidemia, C-reactive protein, N-terminal pro-brain natriuretic peptide, high sensitivity cardiac } \\
\text { troponin-l, estimated glomerular filtration rate, SYNTAX score, statins and anti-diabetes use at } \\
\text { baseline, ticagrelor and low molecular weight heparin use during hospitalization }\end{array}$} \\
\hline
\end{tabular}

\section{Discussion}

To our knowledge, this is the first few studies to evaluate the impact of $L p(a)$ on coronary artery lesion and in-hospital outcomes in ACS patients undergoing PCI in China. There are two main findings of our current analyses: 1 ) increased $L p(a)$ was associated with a more severe coronary artery lesion as reflected by numbers of coronary arteries $\geq 70 \%$ stenosis, type $\mathrm{C}$ coronary lesion and pre-PCI TIME flow grade $1 / 0 ; 2)$ after adjustment for potential covariates, increased $L p(a)$ was associated with a higher risk of congestive heart failure and composite in-hospital outcomes. These findings highlight the importance of measuring $\mathrm{Lp}(\mathrm{a})$ in ACS patients, which might help to improve cardiovascular risk stratification. With respect to the efficacy of PCSK9 inhibitor on Lp(a) reduction, future researches are needed to evaluate whether PCSK9 inhibitor can reduce in-hospital cardiovascular events in ACS patients with high Lp(a) in Chinese populations.

In ACS patients after $\mathrm{PCl}$ treatment, intensive statins has been recommended as the cornerstone treatment. Nonetheless, numerous studies have reported that despite adherence to intensive statins treatment, a substantial proportion of patients remain experience stent thrombosis, myocardial infarction, ischemic stroke/TIA and cardiovascular death. The reasons for the residual cardiovascular risks are likely multifactorial $[7,8,13,18]$, which included systemic inflammation, uncontrolled other risk factors (e.g. 
hypertension and diabetes), poor adherence to antiplatelet medications, and among others. Importantly, prior studies also have shown that increased $L p(a)$ is associated with a variety of cardiovascular diseases such as CHD and congestive heart failure [24-26]. The pathophysiological effects of increased $\mathrm{Lp}(\mathrm{a})$ on cardiovascular systems are two folds, that is pro-atherosclerosis and pro-thrombosis. Through binding to circulating oxidized phospholipid and apoprotein B100 (OxPL/ApoB100), Lp(a) exerts potent inflammatory and oxidative effects on endothelial cells, causing endothelial dysfunction, macrophages migration and proliferation, foams cells accumulation, and necrotic core expansion $[13,23]$. On the other hand, $L p(a)$ also plays an important role in impairing endogenous fibrinolysis, promoting platelet aggregation and thrombosis formation $[13,23,27]$. These two pathophysiological functions of $\operatorname{Lp}(\mathrm{a})$ predispose patients with high $\operatorname{Lp}(\mathrm{a})$ at substantial high residual cardiovascular risks. Indeed, prior studies showed that compared to those with low $\operatorname{Lp}(a)$, high $\operatorname{Lp}(a)$ was associated with more severe coronary artery stenosis as detected by angiography [28], and was also associated with less coronary collateral circulation in patients with acute myocardial infarction [29]. In addition, one study reported that increased $L p(a)$ at baseline was associated with a higher risk of stent restenosis and revascularization [11]. Two additional studies also showed that plasma $L p(a)$ concentration was an independent predictor of stent restenosis [30,31]. Consistent to prior reports, results of our current study also suggest that in ACS patients undergoing $\mathrm{PCl}$, compared to those with low $\mathrm{Lp}(\mathrm{a})$, patients with high $\mathrm{Lp}(\mathrm{a})$ had a higher unadjusted risk of acute stent thrombosis, which might be due to the less optimal post-PCI TIMIE flow in these ACS populations. As shown in Table 4 that high Lp(a) was associated with post-PCI TIMI flow grade $1 / 0$, and after adjustment for glycoprotein Ilb/Illa inhibitor, the association was attenuated into statistical insignificance, suggesting that in ACS patients with high $\operatorname{Lp}(\mathrm{a})$, use of glycoprotein Ilb/IIla inhibitor during $\mathrm{PCl}$ might mitigate the potential thrombotic risk of $\mathrm{Lp}(\mathrm{a})$. Future studies are needed to corroborate our findings.

Few studies have reported that increased $\operatorname{Lp}(\mathrm{a})$ was associated with a higher risk of congestive heart failure in Caucasian populations. For example, Kamstrup et al reported that elevated $L p(a)$ level was associated with an increased risk of congestive heart failure [32]. Steffen et al also found that Lp(a)related risks of congestive heart failure were only evident in Caucasian populations but not in Black or Asian populations [24]. Interestingly and importantly, our current study for the first time showed that compared to those with low $\operatorname{Lp}(a)$, patients with high $\operatorname{Lp}(a)$ had higher risk of congestive heart failure even after adjustment for potential covariates. The underlying mechanisms are likely multifactorial, and we considered that the higher incidence of congestive heart failure might be due to more severe ischemic injury in ACS patients with high $L p(a)$. Indeed, patients with high $L p(a)$ had larger number of coronary stenosis $\geq 70 \%$, were more likely to have type $\mathrm{C}$ lesion and left anterior descending coronary stenosis, and poorer coronary perfusion pre- and post-PCl treatment. Further studies are needed to corroborate our current findings and if confirmed, $L p(a)$ might be used to predict the incidence of congestive heart failure.

There are some limitations of our current study. First, this is a retrospective and observational study, and findings from current study cannot be drawn causal relationship. Second, due to the difference in $L p(a)$ gene expression between different racial/ethnic populations, current findings might not be extrapolated to other populations. Third, although extensive adjustment for potential covariates, unmeasured and 
undetected covariates might still exist and influence the association of $L p(a)$ and outcomes. Fourth, we only evaluated the association of $L p(a)$ and in-hospital outcomes and whether these observations extended to long-term outcome was unknown. Last but not the least, the modest sample size might not be able to find significant association of high $L p(a)$ and other cardiovascular events such as myocardial infarction.

\section{Conclusion}

In conclusion, our current study shows that in ACS patients undergoing $\mathrm{PCl}$, high $\mathrm{Lp}(\mathrm{a})$ was associated with more severe coronary artery lesion. In addition, high $L p(a)$ was also associated with higher risk of congestive heart failure and composite in-hospital outcomes. Future studies are needed to corroborate our current findings, and also needed to evaluate how to mitigate the cardiovascular risk associated with increased $L p(a)$.

\section{Abbreviations}

Coronary heart disease (CHD)

Low-density lipoprotein cholesterol (LDL-C)

Percutaneous coronary intervention (PCl)

Lipoprotein (a) [Lp(a)]

Acute coronary syndrome (ACS)

Transient ischemic stroke (TIA)

Peripheral vascular disease (PVD)

Chronic kidney disease (CKD)

Body mass index (BMI)

Glycated hemoglobin A1c (HbA1c)

C-reactive protein (CRP)

High-sensitivity cardiac troponin-I (Hs-cTNI)

$\mathrm{N}$-terminal pro-B natriuretic peptide (NT-proBNP)

Estimated glomerular filtration rate (eGFR)

Standard deviation (SD) 
Interquartile range (IQR)

\section{Declarations}

\section{Ethics approval and consent to participate}

The study design was approved by the Clinical and Basic Research Ethic Committee of Fuwai Hospital. This was a retrospective study and no written informed consent was required.

\section{Consent for publication}

Not Applicable.

\section{Availability of data and materials}

The datasets used and/or analysed during the current study available from the corresponding author on reasonable request.

\section{Competing interests}

None.

\section{Funding}

Not Applicable.

\section{Author Contributions}

BQU and FFZ conceived the study, participated in the design and drafted the manuscript; BQW and HJZ performed the statistical analyses; BQW, HJZ, CHL, HL, RSL, JL, ZLJ and FFZ collected the data. All authors read and approved the final manuscript.

\section{Acknowledgement}

We appreciate very much for our colleagues who help to adjudicate the clinical outcomes.

\section{References}


1. Virani SS, Alonso A, Benjamin EJ, Bittencourt MS, Callaway CW, Carson AP, Chamberlain AM, Chang AR, Cheng S, Delling FN et al: Heart Disease and Stroke Statistics-2020 Update: A Report From the American Heart Association. Circulation 2020, 141(9):e139-e596.

2. [Chinese guidelines for the prevention of cardiovascular diseases(2017)]. Zhonghua xin xue guan bing za zhi 2018, 46(1):10-25.

3. Smith SC, Jr., Benjamin EJ, Bonow RO, Braun LT, Creager MA, Franklin BA, Gibbons RJ, Grundy SM, Hiratzka LF, Jones DW et al: AHAVACCF secondary prevention and risk reduction therapy for patients with coronary and other atherosclerotic vascular disease: 2011 update: a guideline from the American Heart Association and American College of Cardiology Foundation endorsed by the World Heart Federation and the Preventive Cardiovascular Nurses Association. Journal of the American College of Cardiology 2011, 58(23):2432-2446.

4. Mach F, Baigent C, Catapano AL, Koskinas KC, Casula M, Badimon L, Chapman MJ, De Backer GG, Delgado V, Ference BA et al: 2019 ESC/EAS Guidelines for the management of dyslipidaemias: lipid modification to reduce cardiovascular risk. European heart journal2020, 41(1):111-188.

5. Yusuf S, Rangarajan S, Teo K, Islam S, Li W, Liu L, Bo J, Lou Q, Lu F, Liu T et al: Cardiovascular risk and events in 17 low-, middle, and high-income countries. The New England journal of medicine 2014, 371(9):818-827.

6. Ferrari R, Aguiar C, Alegria E, Bonadonna RC, Cosentino F, Elisaf M, Farnier M, Ferrieres J, Filardi PP, Hancu $\mathrm{N}$ et al: Current practice in identifying and treating cardiovascular risk, with a focus on residual risk associated with atherogenic dyslipidaemia. European heart journal supplements : journal of the European Society of Cardiology 2016, 18(Suppl C):C2-c12.

7. Nichols GA, Philip S, Reynolds K, Granowitz CB, Fazio S: Increased residual cardiovascular risk in patients with diabetes and high versus normal triglycerides despite statin-controlled LDL cholesterol. Diabetes, obesity \& metabolism 2019, 21(2):366-371.

8. Lieb W, Enserro DM, Larson MG, Vasan RS: Residual cardiovascular risk in individuals on lipidlowering treatment: quantifying absolute and relative risk in the community. Open heart 2018, 5(1):e000722.

9. Saleheen D, Haycock PC, Zhao W, Rasheed A, Taleb A, Imran A, Abbas S, Majeed F, Akhtar S, Qamar N et al: Apolipoprotein(a) isoform size, lipoprotein(a) concentration, and coronary artery disease: a mendelian randomisation analysis. The lancet Diabetes \& endocrinology 2017, 5(7):524-533.

10. Verbeek R, Hoogeveen RM, Langsted A, Stiekema LCA, Verweij SL, Hovingh GK, Wareham NJ, Khaw KT, Boekholdt SM, Nordestgaard BG et al: Cardiovascular disease risk associated with elevated lipoprotein(a) attenuates at low low-density lipoprotein cholesterol levels in a primary prevention setting. European heart journal 2018, 39(27):2589-2596.

11. Cai A, Li L, Zhang Y, Mo Y, Li Z, Mai W, Zhou Y: Baseline LDL-C and Lp(a) elevations portend a high risk of coronary revascularization in patients after stent placement. Disease markers 2013, 35(6):857-862. 
12. Nordestgaard BG, Langsted A: Lipoprotein (a) as a cause of cardiovascular disease: insights from epidemiology, genetics, and biology. Journal of lipid research 2016, 57(11):1953-1975.

13. Cai A, Li L, Zhang Y, Mo Y, Mai W, Zhou Y: Lipoprotein(a): a promising marker for residual cardiovascular risk assessment. Disease markers 2013, 35(5):551-559.

14. Willeit P, Ridker PM, Nestel PJ, Simes J, Tonkin AM, Pedersen TR, Schwartz GG, Olsson AG, Colhoun $\mathrm{HM}$, Kronenberg $\mathrm{F}$ et al: Baseline and on-statin treatment lipoprotein(a) levels for prediction of cardiovascular events: individual patient-data meta-analysis of statin outcome trials. Lancet (London, England) 2018, 392(10155):1311-1320.

15. Burgess S, Ference BA, Staley JR, Freitag DF, Mason AM, Nielsen SF, Willeit P, Young R, Surendran P, Karthikeyan $S$ et al: Association of LPA Variants With Risk of Coronary Disease and the Implications for Lipoprotein(a)-Lowering Therapies: A Mendelian Randomization Analysis. JAMA cardiology 2018, 3(7):619-627.

16. Gazzola K, Reeskamp L, van den Born BJ: Ethnicity, lipids and cardiovascular disease. Current opinion in lipidology 2017, 28(3):225-230.

17. O'Donoghue ML, Fazio S, Giugliano RP, Stroes ESG, Kanevsky E, Gouni-Berthold I, Im K, Lira Pineda A, Wasserman SM, Češka R et al: Lipoprotein(a), PCSK9 Inhibition, and Cardiovascular Risk. Circulation 2019, 139(12):1483-1492.

18. Pradhan AD, Aday AW, Rose LM, Ridker PM: Residual Inflammatory Risk on Treatment With PCSK9 Inhibition and Statin Therapy. Circulation 2018, 138(2):141-149.

19. Cutlip DE, Windecker S, Mehran R, Boam A, Cohen DJ, van Es GA, Steg PG, Morel MA, Mauri L, Vranckx $\mathrm{P}$ et al: Clinical end points in coronary stent trials: a case for standardized definitions. Circulation 2007, 115(17):2344-2351.

20. Thygesen K, Alpert JS, Jaffe AS, Chaitman BR, Bax JJ, Morrow DA, White HD: Fourth Universal Definition of Myocardial Infarction (2018). Journal of the American College of Cardiology 2018, 72(18):2231-2264.

21. Powers WJ, Rabinstein AA, Ackerson T, Adeoye OM, Bambakidis NC, Becker K, Biller J, Brown M, Demaerschalk BM, Hoh B et al: 2018 Guidelines for the Early Management of Patients With Acute Ischemic Stroke: A Guideline for Healthcare Professionals From the American Heart Association/American Stroke Association. Stroke 2018, 49(3):e46-e110.

22. Yancy CW, Jessup M, Bozkurt B, Butler J, Casey DE, Jr., Drazner MH, Fonarow GC, Geraci SA, Horwich T, Januzzi JL et al: 2013 ACCF/AHA guideline for the management of heart failure: executive summary: a report of the American College of Cardiology Foundation/American Heart Association Task Force on practice guidelines. Circulation 2013, 128(16):1810-1852.

23. Nordestgaard BG, Chapman MJ, Ray K, Boren J, Andreotti F, Watts GF, Ginsberg H, Amarenco P, Catapano A, Descamps OS et al: Lipoprotein(a) as a cardiovascular risk factor: current status. European heart journal 2010, 31(23):2844-2853.

24. Steffen BT, Duprez D, Bertoni AG, Guan W, Tsai MY: Lp(a) [Lipoprotein(a)]-Related Risk of Heart Failure Is Evident in Whites but Not in Other Racial/Ethnic Groups. Arteriosclerosis, thrombosis, and 
vascular biology 2018, 38(10):2498-2504.

25. Tsimikas S, Karwatowska-Prokopczuk E, Gouni-Berthold I, Tardif JC, Baum SJ, Steinhagen-Thiessen E, Shapiro MD, Stroes ES, Moriarty PM, Nordestgaard BG et al: Lipoprotein(a) Reduction in Persons with Cardiovascular Disease. The New England journal of medicine 2020, 382(3):244-255.

26. Waldeyer C, Makarova N, Zeller T, Schnabel RB, Brunner FJ, Jørgensen T, Linneberg A, Niiranen T, Salomaa V, Jousilahti $\mathrm{P}$ et al: Lipoprotein(a) and the risk of cardiovascular disease in the European population: results from the BiomarCaRE consortium. European heart journal 2017, 38(32):24902498.

27. Boffa MB, Koschinsky ML: Lipoprotein (a): truly a direct prothrombotic factor in cardiovascular disease? Journal of lipid research 2016, 57(5):745-757.

28. Lima LM, Carvalho M, Loures-Vale AA, Fernandes AP, Mota AP, Neto CP, Garcia JC, Saad JA, Souza Mde O: Increased serum levels of lipoprotein(a) correlated with the severity of coronary artery disease in patients submitted to angiography. Arquivos brasileiros de cardiologia 2006, 87(3):260266.

29. You XD, Jin JL, Zhang H, Guo N, Hou BJ, Guo YL, Wu NQ, Zhu CG, Li JJ: Lipoprotein(a) as a marker for predicting coronary collateral circulation in patients with acute myocardial infarction. Personalized medicine 2020, 17(1):67-78.

30. Kamitani T, Taniguchi T, Miyai N, Kawasaki T, Kawasaki S, Sugihara H: Association between plasma lipoprotein(a) concentration and restenosis after stent implantation. Circulation journal : official journal of the Japanese Circulation Society 2005, 69(6):644-649.

31. Qin SY, Liu J, Jiang HX, Hu BL, Zhou Y, Olkkonen VM: Association between baseline lipoprotein (a) levels and restenosis after coronary stenting: meta-analysis of 9 cohort studies. Atherosclerosis 2013, 227(2):360-366.

32. Kamstrup PR, Nordestgaard BG: Elevated Lipoprotein(a) Levels, LPA Risk Genotypes, and Increased Risk of Heart Failure in the General Population. JACC Heart failure 2016, 4(1):78-87.

\section{Figures}


A total of 3108 CHD patients were screened between January

of 2017 and December of 2019

1425 patients were excluded due to not present as acute coronary syndrome

1683 patients were presented as ACS

391 patients were excluded due to following: 82 underwent CABG, 184 critical ill; 67 failed to have coronary stenting; 58 died before PCI

1292 ACS patients were included into final analysis

Figure 1

Figure 1

Study flowchart 